\title{
Viscosity Dependence of a Magnetic Fluid Nanoparticles Concentration
}

\author{
J. Tóthováa ${ }^{a}$, J. Kováč ${ }^{b}$, P. KopČAnskÝ ${ }^{b}$, M. RajñáK ${ }^{b}$, K. PauloviČOvá ${ }^{b}$, \\ M. TimKO ${ }^{b, *}$ AND A. JózEFCZAK ${ }^{c}$ \\ ${ }^{a}$ Department of Physics, Faculty of Electrical Engineering and Informatics, Technical University of Košice, \\ Park Komenského 2, 04200 Košice, Slovakia \\ ${ }^{b}$ Institute of Experimental Physics SAS, Watsonova 47, 04001 Košice, Slovakia \\ ${ }^{c}$ Institute of Acoustics, Faculty of Physics, Adam Mickiewicz University, Umultowska 85, 61614 Poznań, Poland

\begin{abstract}
In this work we have studied the effect of temperature on the viscosity of magnetic fluids (MFs) based on the transformer oil ITO 100. The volume concentration of suspended magnetic particles (MPs) changed from 0.25 to $1 \%$. Rheological characterization of MFs was performed using a vibroviscometer at working frequency $50{ }^{\circ} \mathrm{C}$. The magnetization of different concentrations of MPs in MFs was determined by using the vibrating sample magnetometer.
\end{abstract} \\ of $30 \mathrm{~Hz}$. The temperature dependence of the viscosity was measured in the temperature range from 20 up to
}

DOI: $10.12693 /$ APhysPolA.126.278

PACS: 07.55.Jg, 75.50.Mm, 83.85.Jn

\section{Introduction}

Ferrofluids or magnetic fluids (MFs) are colloidal suspensions of nanosized ferromagnetic particles coated with surfactants and dispersed in a carrier liquid. Since their physical properties can be easily influenced by external forces such as the magnetic field, they have found many applications in a variety of fields such as electronic packing, mechanical engineering, aerospace or bioengineering. In particular, if MFs are based on water as a carrier liquid, they can be used in such areas as magnetic drug delivery, cancer treatment by means of magnetic induced hyperthermia, or magnetic imaging [1]. One of many unique properties of ferrofluids is their tunable viscosity by the external magnetic field. This is called the magnetoviscous effect $[2,3,4]$. One of the most successful applications of thermophysical property of magnetic fluid is audio speaker. In the audio speakers, magnetic fluid is filled around the voice coil. Because thermal conductivity of magnetic fluid is much larger than that of the air, the fluid provides a lower heat resistance between the coil and pole plate [5].

However, if we apply a magnetic fluid to heat transfer applications such as cooling system for power transformer or micromachine [6], it is necessary to perform the detailed investigation on the properties of heat transfer of a magnetic fluid under magnetic field. Several studies on the heat transfer characteristics of magnetic fluid have been carried out for a long time with great engineering interest [7]. However, the heat transfer phenomena of a magnetic fluid are very complicated and there is not

*corresponding author; e-mail: timko@saske.sk enough knowledge about it. For a good understanding of this phenomenon, the knowledge of the viscous properties of MFs in the absence of magnetic fields is very important. Particularly its dependence on the amount of suspended magnetic particles (MPs) and temperature is very interesting.

\section{Experiment}

In the present work, MFs based on inhibited oil ITO 100 as carrier liquid was used. The stock MF was prepared by the chemical precipitation method. To stabilize the suspensions, oleic acid was selected as the activator to cover the nanoparticles. The amount of activator was calculated with weight percentage of the suspensions of magnetic $\left(\mathrm{Fe}_{3} \mathrm{O}_{4}\right)$ nanoparticles and oil. The samples with different particle volume fractions $\phi$ (0.25 sample MF1, 0.50 sample MF2 and $1.00 \%$ sample MF3) were obtained by diluting the stock MF solution. Rheological characterization of MFs was performed by a Sine-wave Vibro Viscometer SV-10, featuring the vibrating tuning fork measurement method. It measures viscosity by detecting the driving electric current necessary to resonate two sensor plates at constant frequency of $30 \mathrm{~Hz}$ and amplitude of less than $1 \mathrm{~mm}$. The temperature dependence of the viscosity was measured in the temperature range from 20 to $50{ }^{\circ} \mathrm{C}$. Magnetization properties of the prepared MFs were determined by vibrating sample magnetometer (VSM) at room temperature.

\section{Results and discussion}

The saturation magnetization at room temperature of individual samples increases with increasing amount of MPs in MFs as it can be seen in Fig. 1. The obtained values of saturation magnetization were $0.4,1.6$ 
and $3.5 \mathrm{emu} / \mathrm{g}$ for sample MF1, MF2 and MF3, respectively.

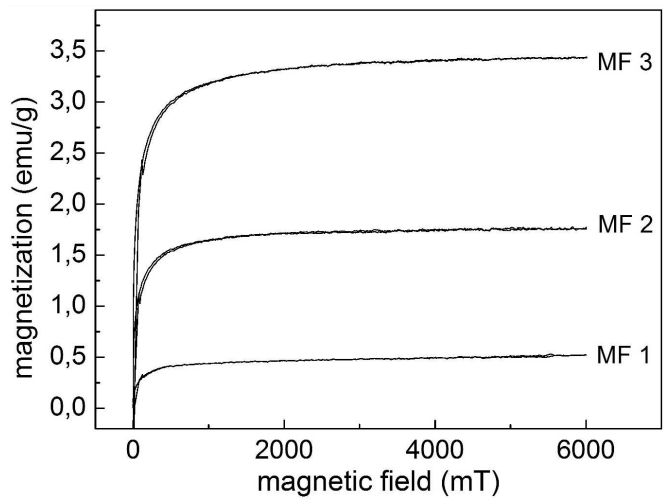

Fig. 1. Magnetization dependence of four different MFs on applied magnetic field. MF 1,2, 3 correspond to volume concentrations $0.25,0.50$ and $1.00 \%$, respectively.

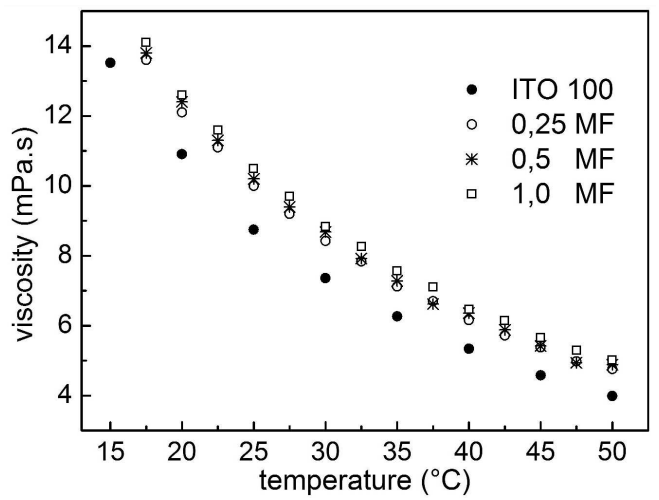

Fig. 2. The viscosity versus increasing temperature for $0.25,0.5$ and $1.0 \%$ MFs in ITO 100 oil as the carrier liquid.

As it was shown earlier [8], the shear stress versus shear rate dependence for pure transformer oil ITO 100 is linear, which corresponds to a Newtonian-like fluid behavior. Similarly prepared samples of magnetic fluids based on this oil and used in our experiment behave as Newtonian fluids, since their viscosities do not depend on the shear rate too (results are not given here). The temperature dependence of viscosity (Fig. 2) shows the classical behaviour, at which the increasing temperature initiates decrease of viscosity for all volume concentrations of magnetite in the samples.

The obtained experimental data were fitted with the Arrhenius type formula [9]:

$$
\eta=\eta_{\text {ref }} \exp \left[\frac{E_{a}}{R}\left(\frac{1}{T}-\frac{1}{T_{\text {ref }}}\right)\right] .
$$

Here $R=8.314 \mathrm{~J} / \mathrm{mol} \cdot \mathrm{K}$ is the universal gas constant, $E_{a}$ is the viscous flow activation energy, $T_{r e f}$ is the reference temperature $293.15 \mathrm{~K}, \eta_{\text {ref }}$ the viscosity at $T_{\text {ref }}$, and $\eta_{0}$ - that of carrier liquid. The calculated values of $E_{a}$ and the volume concentrations of magnetic particles $\phi$ with corresponding saturation magnetizations $M_{s}$ are summarized in Table.

The influence on viscosity arises from the change of the viscosity of pure oil with temperature and from Brownian motion of nanoparticles [10]. With the increasing temperature the Brownian motion of the particles in the FFs was strengthened, which reduced the speed difference between the carrier liquid and the magnetic particles.

TABLE

Characteristics of investigated samples.

\begin{tabular}{c|c|c|c|c}
\hline \hline Sample & $\begin{array}{c}M_{s} \\
(\mathrm{emu} / \mathrm{g})\end{array}$ & $\begin{array}{c}\phi \\
(\%)\end{array}$ & $\begin{array}{c}\eta / \eta_{0\left(25^{\circ} \mathrm{C}\right)} \\
(\mathrm{mPa} \cdot \mathrm{s})\end{array}$ & $\begin{array}{c}E_{a} \cdot 10^{-4} \\
(\mathrm{~J} / \mathrm{mol})\end{array}$ \\
\hline ITO 100 & 0 & 0 & 1 & 2.758 \\
\hline ITO MF1 & 0.4 & 0.25 & 1.14 & 2.568 \\
\hline ITO MF2 & 1.6 & 0.50 & 1.17 & 2.544 \\
\hline ITO MF3 & 3.5 & 1.00 & 1.20 & 2.528
\end{tabular}

\section{Conclusions}

In the presented experiments, the temperature and concentration dependencies of the viscosity of MFs, as colloidal suspensions of magnetic particles on the base of ITO 100 oil, have been measured. Our measurements confirm the expected behaviour of MFs, for which the viscosity increases with concentration of magnetite nanoparticles and decreases with the increase of temperature for all samples.

\section{Acknowledgments}

This work was supported by the Ministry of Education Agency for the Structural Funds of the EU within the projects NFP 26220120021, 26220120033, 26110230061, 26220120046 and by the grant VEGA $1 / 0370 / 12$ and $2 / 0043 / 2012$ and by a Polish National Science Centre grant, no DEC-2011/03/B/ST7/00194.

\section{References}

[1] Q.A. Pankhurst, N.K.T. Thanh, S.K. Jones, J. Dobson, J. Phys. D: Appl. Phys. 42, 224001 (2009).

[2] S. Odenbach, Magnetoviscous Effects in Ferrofluids ISBN 3-540-43068-7, Springer-Verlag, Berlin, 2002.

[3] Y.Yamada, Y. Enomoto, Physica A 387, 1 (2008).

[4] D. Soto-Aquino, C. Rinaldi, J. Magn. Mag. Mat. 323, 1319 (2011).

[5] D.B. Hathaway, Sound Eng. Mag. 13, 42 (1979).

[6] K. Nakatsuka, B. Jeyadevan, S. Neveu, H. Koganezawa, J. Magn. Mag. Mat. 252, 360 (2002).

[7] L.S. Sundar, M.K. Singh, A.C.M. Sousa, Int. Commun. Heat Mass Transfer 44, 7 (2013).

[8] A. Józefczak, T. Hornowski, A. Skumiel, Int. J. Thermophys 32, 795 (2011).

[9] T.G. Mezger, The Rheology Handbook, ISBN 386630-864-7, Vincentz Network, Hanover 2011. Int. Communications in Heat and MassTransfer.

[10] S. Wang, Ch. Yang, X. Bian, J. Magn. Mag. Mat. 324, 3361 (2012). 